\title{
Phase Algorithm for Blocking Artifact Reduction in Reconstructions from Analysis-Only AM-FM Models
}

\author{
Krishnapraveen Suri and Joseph P. Havlicek \\ School of Electrical and Computer Engineering, University of Oklahoma, Norman, OK 73019 USA
}

\begin{abstract}
Virtually all techniques for computing AM-FM models involve jointly localized filterbanks and nonlinear approximations that preclude the possibility of perfect reconstruction. Although perfect reconstruction models have begun to emerge, they are currently of limited practical interest because of their large complexity. Thus, it is of interest to obtain approximate reconstructions from the analysis-only models because these provide insight into the accuracy of the AM and FM functions. However, the only existing 2-D reconstruction algorithm suffers from severe blocking artifacts that limit its usefulness in this regard. In this paper we present a new reconstruction algorithm that makes full use of all available phase reconstruction boundary conditions in a multipath interpolative scheme to eliminate the blocking artifacts, thereby dramatically improving the utility of the approximate reconstructions.
\end{abstract}

\section{Introduction}

AM-FM models represent images directly in terms of spatially and spectrally localized amplitude and frequency modulations consistent with certain processes known to occur in the early stages of biological vision systems. Since their utility in performing texture segmentation was first demonstrated in $[1,2]$, these models have been used in a variety of texture analysis applications [6], where performance has generally been improved by utilizing explicitly computed modulations as opposed to features such as Gabor filter response magnitudes and their simple moments, which may be interpreted as coarsely approximating the true modulations. Here, we restrict our attention to complex valued models of the form

$$
t(\mathbf{x})=\sum_{k=1}^{K} t_{k}(\mathbf{x})=\sum_{k=1}^{K} a_{k}(\mathbf{x}) \exp \left[j \varphi_{k}(\mathbf{x})\right]
$$

where the image $t(\mathbf{x})$ is considered as a sum of AMFM components; real valued images may be analyzed

This work was supported in part by the U.S. Army Research Laboratory and the U.S. Army Research Office under grant W911NF-04-1-0221. against (1) using the complex extension described in [5, 6]. For each component $t_{k}$, the FM function $\nabla \varphi_{k}$ characterizes the local texture orientation and granularity, while the AM function $a_{k}$ captures the local texture contrast. Collectively, these functions provide a rich, powerful modulation domain description of the image useful for constructing potent, biologically motivated feature vectors.

Practical algorithms for estimating $a_{k}$ and $\nabla \varphi_{k}$ in (1) were given in $[5,8]$. Algorithms of this type suffer from two main limitations. First, due to their nonlinearity, they cannot be applied directly to a sum of components as in (1). Thus, it is necessary to isolate the components $t_{k}$ on a spatially local basis prior to demodulation. Linear filterbanks are almost always used for this purpose. Second, all algorithms of this type are based on approximations that break down when applied to a signal that is not locally narrowband. The general strategy that has emerged for working within these limitations is to first process the image with a multiband filterbank to isolate narrowband components, and then estimate the AM and FM functions of each component by demodulating the filterbank channel responses.

The filters must possess a high degree of spatio-spectral localization; otherwise, locally narrowband components will not generally be obtained and the estimated AM and FM functions will be characterized by large approximation errors. Hence, Gabor and related filters have been popular. However, good joint localization and finite support simultaneously in space and frequency are conflicting design goals. Thus, these filters can provide neither orthogonality nor perfect reconstruction. Nevertheless, such AM-FM models have proven valuable in analysis-only applications such as segmentation. New techniques for computing perfect reconstruction AM-FM models have emerged recently $[6,10]$, but require an order of magnitude more components $t_{k}$ than their analysis-only counterparts. Thus analysis-only models are important in practice, where approximate AM-FM image reconstructions are of interest because they provide an indication of how well the approximations in the demodulation algorithms are holding up.

Reconstruction of the phase $\varphi_{k}$ from samples of the estimated frequency field $\nabla \varphi_{k}$ is a difficult, ill-posed inverse 
problem. Approximate 1-D reconstruction was demonstrated in $[3,7]$. In $2-\mathrm{D}$, the only previous algorithm is the unsophisticated approach given in $[4,5]$, which reconstructs the phase independently on regularly sized blocks in order to limit error propagation. The reconstruction process is re-initialized at the upper left corner of each block using a phase boundary condition that is saved as part of the AM-FM model. As should be expected, this tends to introduce strong blocking artifacts that limit the usefulness of the reconstructions for assessing the quality of the estimated modulations. Moreover, the information available in nearby boundary conditions from adjacent blocks goes underutilized. In this paper, we present a new 2-D phase algorithm that provides smooth, seamless reconstruction across block boundaries by fully utilizing all available boundary conditions in a multipath interpolative reconstruction scheme.

\section{Phase Reconstruction}

Let $\mathbf{x}=[x y]^{T} \in \mathbb{Z}^{2}, \mathbf{e}_{1}=\left[\begin{array}{ll}1 & 0\end{array}\right]^{T}$, and $\mathbf{e}_{2}=\left[\begin{array}{ll}0 & 1\end{array}\right]^{T}$. Let $t(\mathbf{x}): \mathbb{Z}^{2} \rightarrow \mathbb{C}$ and assume that a $K$-component AM-FM model $\left\{a_{k}(\mathbf{x}), \nabla \varphi_{k}(\mathbf{x})\right\}_{k \in[1, K]}$ has been obtained. Moreover, assume that estimates $\phi_{p}$ of the phase $\varphi_{k}$ of each component have been saved as side information on a $B C \times B C$ pixel sublattice, where typically $2 \leq B C \leq 64$.

Often, the filterbank channels have conjugate symmetric impulse responses $g_{k}$ and hence real-valued frequency responses $G_{k}$; the quasi-eigenfunction approximation of [5] then implies that the channel response $y_{k}$ is approximately

$y_{k}(\mathbf{x})=t(\mathbf{x}) * g_{k}(\mathbf{x}) \approx t_{k}(\mathbf{x}) * g_{k}(\mathbf{x}) \approx G_{k}\left[\nabla \varphi_{k}(\mathbf{x})\right] t_{k}(\mathbf{x})$,

where $t_{k}$ is the component in (1) that dominates $y_{k}$ in a neighborhood about the pixel $\mathbf{x}$. Thus, on the sublattice, phase boundary conditions $\phi_{p}$ for $t_{k}$ may be set equal to the channel phase $\arg y_{k}$, which approximates $\varphi_{k}$.

Reconstruction of the image according to (1) is straightforward once the phases are obtained for all $K$ components. Hence, the central problem we consider is that of reconstructing $\varphi_{k}$ given the phase boundary counditions and the estimated frequency field $\nabla \varphi_{k}$. The situation is depicted in Fig. 1, where circles denote pixel sites and $B C=3$. Phase reconstruction is carried out independently on $B C \times B C$ blocks to limit error propagation, and four adjacent blocks are shown. The gray circles denote sublattice sites where boundary conditions $\left\{\varphi_{p}(\mathbf{x})\right\}_{p \in[1,4]}$ have been saved.

With the previous algorithm of $[4,5]$, phase reconstruction on Block-1 is initialized to $\phi_{1}$ in the upper left corner. Horizontal frequency estimates $\mathbf{e}_{1}^{T} \nabla \varphi_{k}$ are accumulated to reconstruct $\varphi_{k}$ on the first row of the block, while vertical frequencies $\mathbf{e}_{2}^{T} \nabla \varphi_{k}$ are accumulated to reconstruct the first column. On the interior of the block, the horizontal and vertical estimates are averaged according to

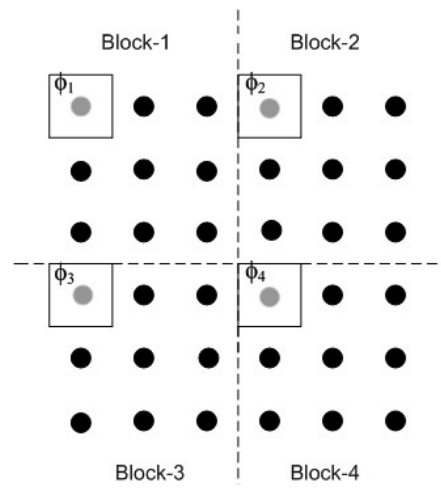

Figure 1. Four adjacent phase reconstruction blocks and their associated phase boundary conditions $\phi_{1}$ through $\phi_{4}$.

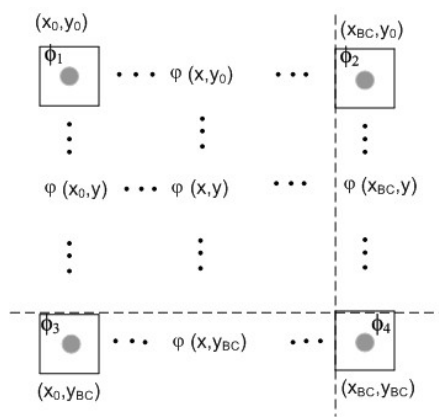

Figure 2. Coordinate system for calculating the phase estimates $\varphi_{1}(\mathrm{x})$ through $\varphi_{4}(\mathrm{x})$.

$\varphi_{k}(\mathbf{x})=\frac{1}{2}\left\{\left[\varphi_{k}\left(\mathbf{x}-\mathbf{e}_{1}\right)+\mathbf{e}_{1}^{T} \nabla \varphi_{k}\left(\mathbf{x}-\mathbf{e}_{1}\right)\right]+\left[\varphi_{k}(\mathbf{x}-\right.\right.$ $\left.\left.\left.\mathbf{e}_{2}\right)+\mathbf{e}_{2}^{T} \nabla \varphi_{k}\left(\mathbf{x}-\mathbf{e}_{2}\right)\right]\right\}$. The advantages of this approach are that it is simple and does not require phase unwrapping of the boundary conditions. The disadvantages are that it fails to utilize the information in $\phi_{2}, \phi_{3}$, and $\phi_{4}$ and that it produces severe blocking artifacts.

\section{The New Algorithm}

We present a new phase algorithm that utilizes all four boundary conditions $\left\{\phi_{p}\right\}_{p \in[1,4]}$ to reconstruct $\varphi_{k}$ on Block-1. This improves the quality of the reconstruction and virtually eliminates the blocking artifacts. However, because multiple boundary conditions are used within each block, phase unwrapping of the boundary conditions is required. We perform unwrapping on a full resolution phase image using the algorithm of [9]. The unwrapped phase is sampled on the sublattice to obtain the conditions $\phi_{p}$, which are saved as part of the computed AM-FM model.

For each pixel $\mathbf{x}$, four phase estimates $\left\{\varphi_{p}(\mathbf{x})\right\}_{p \in[1,4]}$ 
are obtained by accumulating frequency estimates $\nabla \varphi_{k}(\mathbf{x})$ along a path connecting $\phi_{p}$ to the pixel site $\mathbf{x}$, where $\left\|\phi_{p} \rightarrow \varphi(\mathbf{x})\right\|$ denotes the path length. These four estimates are then interpolated using weights inversely proportional to the path length according to

$$
\varphi_{k}(\mathbf{x})=\frac{\sum_{p=1}^{4}\left\|\phi_{p} \rightarrow \varphi(\mathbf{x})\right\|^{-1} \varphi_{p}(\mathbf{x})}{\sum_{p=1}^{4}\left\|\phi_{p} \rightarrow \varphi(\mathbf{x})\right\|^{-1}} .
$$

Calculations for $\varphi_{p}$ are given below with reference to the coordinates shown in Fig. 2.

Calculation for $\varphi_{1}(\mathrm{x})$ : Along the top row of Block-1, $\varphi_{1}$ is obtained by accumulation according to

$$
\varphi_{1}\left(x, y_{0}\right)=\phi_{1}+\sum_{m=x_{0}}^{x-1} \mathbf{e}_{1}^{T} \nabla \varphi_{k}\left(m, y_{0}\right) .
$$

Likewise, along the first column of Block-1, we have

$$
\varphi_{1}\left(x_{0}, y\right)=\phi_{1}+\sum_{m=y_{0}}^{y-1} \mathbf{e}_{2}^{T} \nabla \varphi_{k}\left(x_{0}, m\right) .
$$

At the remaining pixels of the block, we take

$$
\begin{aligned}
\varphi_{1}(x, y) & =\frac{1}{2}\left(\varphi_{1}\left(x_{0}, y\right)+\sum_{m=x_{0}}^{x-1} \mathbf{e}_{1}^{T} \nabla \varphi_{k}(m, y)\right. \\
& \left.+\varphi_{1}\left(x, y_{0}\right)+\sum_{m=y_{0}}^{y-1} \mathbf{e}_{2}^{T} \nabla \varphi_{k}(x, m)\right) .
\end{aligned}
$$

Calculation for $\varphi_{\mathbf{2}}(\mathrm{x})$ : We take

$$
\varphi_{2}\left(x, y_{0}\right)=\phi_{2}-\sum_{m=x}^{x_{B C}-1} \mathbf{e}_{1}^{T} \nabla \varphi\left(m, y_{B C}\right)
$$

along the first row of Block-1. Along the first column, we take

$$
\varphi_{2}\left(x_{B C}, y\right)=\phi_{2}+\sum_{m=y_{0}}^{y-1} \mathbf{e}_{2}^{T} \nabla \varphi\left(x_{B C}, m\right) .
$$

At the remaining interior pixels of Block-1 not located in the first column, $\varphi_{2}(\mathbf{x})$ is then calculated according to

$$
\begin{aligned}
\varphi_{2}(x, y) & =\frac{1}{2}\left(\varphi_{2}\left(x_{B C}, y\right)-\sum_{m=x}^{x_{B C}-1} \mathbf{e}_{1}^{T} \nabla \varphi_{k}(m, y)\right. \\
& \left.+\varphi_{2}\left(x, y_{0}\right)+\sum_{m=y_{0}}^{y-1} \mathbf{e}_{2}^{T} \nabla \varphi_{k}(x, m)\right)
\end{aligned}
$$

Finally, as shown in the top two parts of Fig. 3, on the first column of Block-1 $\varphi_{2}(\mathbf{x})$ is given by

$$
\begin{gathered}
\varphi_{2}\left(x_{0}, y\right)=\frac{1}{2}\left(\varphi_{2}\left(x_{B C}, y\right)-\sum_{m=x_{0}}^{x_{B C}-1} \mathbf{e}_{1}^{T} \nabla \varphi_{k}(m, y)\right. \\
\left.+\varphi_{2}\left(x_{0}+1, y_{0}\right)+\Delta_{y}\right)
\end{gathered}
$$
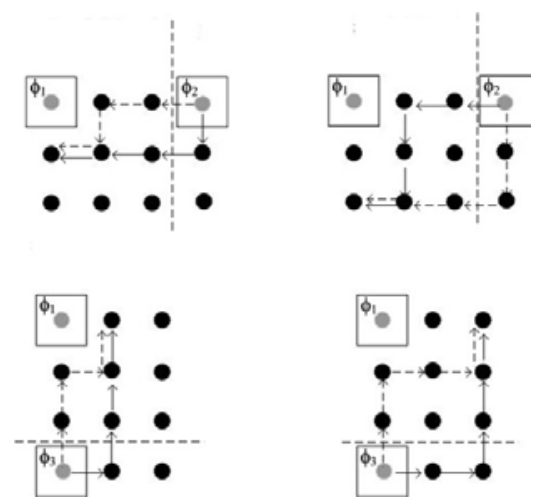

Figure 3. Phase reconstruction paths for (10) (top) and (14) (bottom).

where $\Delta_{y}=\sum_{m=y_{0}}^{y-1} \mathbf{e}_{2}^{T} \nabla \varphi\left(x_{0}+1, m\right)-\mathbf{e}_{1}^{T} \nabla \varphi\left(x_{0}, y\right)$. Calculation for $\varphi_{\mathbf{3}}(\mathrm{x})$ : Along the first column of Block-1, the estimate $\varphi_{3}(\mathbf{x})$ is given by

$$
\varphi_{3}\left(x_{0}, y\right)=\phi_{3}-\sum_{m=y}^{y_{B C}-1} \mathbf{e}_{2}^{T} \nabla \varphi_{k}\left(x_{0}, m\right),
$$

while along the top row of Block-3 it is given by

$$
\varphi_{3}\left(x, y_{B C}\right)=\phi_{3}+\sum_{m=x_{0}}^{x-1} \mathbf{e}_{1}^{T} \nabla \varphi_{k}\left(m, y_{B C}\right)
$$

For interior pixels not on the first row of Block-1, we take

$$
\begin{aligned}
\varphi_{3}(x, y) & =\frac{1}{2}\left(\varphi_{3}\left(x_{0}, y\right)+\sum_{m=x_{0}}^{x-1} \mathbf{e}_{1}^{T} \nabla \varphi_{k}(m, y)\right. \\
& \left.+\varphi_{3}\left(x, y_{B C}\right)-\sum_{m=y}^{y_{B C}-1} \mathbf{e}_{2}^{T} \nabla \varphi_{k}(x, m)\right)
\end{aligned}
$$

The bottom two parts of Fig. 3 depict the paths used for the $\varphi_{3}(\mathbf{x})$ calculation on the first row of Block-1, which is

$$
\begin{gathered}
\varphi_{3}\left(x, y_{0}\right)=\frac{1}{2}\left(\varphi_{3}\left(x, y_{B C}\right)-\sum_{m=y_{0}}^{y_{B C}-1} \mathbf{e}_{2}^{T} \nabla \varphi_{k}(x, m)\right. \\
\left.+\varphi_{3}\left(x_{0}, y_{0}+1\right)+\Delta_{x}\right)
\end{gathered}
$$

where $\Delta_{x}=\sum_{m=x_{0}}^{x-1} \mathbf{e}_{1}^{T} \nabla \varphi\left(m, y_{0}+1\right)-\mathbf{e}_{2}^{T} \nabla \varphi\left(x, y_{0}\right)$.

Calculation for $\varphi_{\mathbf{4}}(\mathrm{x})$ : Along the top row of Block-3 and the first column of Block-2, $\varphi_{4}(\mathbf{x})$ is given, respectively, by

$$
\varphi_{4}\left(x, y_{B C}\right)=\phi_{4}-\sum_{m=x}^{x_{B C}-1} \mathbf{e}_{1}^{T} \nabla \varphi\left(m, y_{B C}\right)
$$



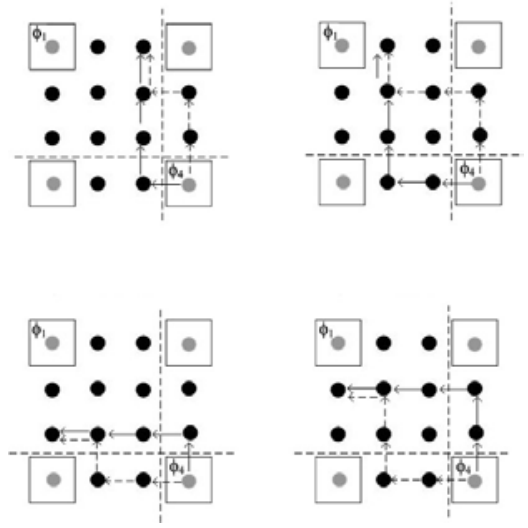

\section{Figure 4. Phase reconstruction paths for (18) (top) and (19) (bottom).}

and

$$
\varphi_{4}\left(x_{B C}, y\right)=\phi_{4}-\sum_{m=y}^{y_{B C}-1} \mathbf{e}_{2}^{T} \nabla \varphi\left(x_{B C}, m\right) .
$$

For the remainder of the pixels that are in neither the first row nor the first column of Block-1, we take

$$
\begin{aligned}
\varphi_{4}(x, y) & =\frac{1}{2}\left(\varphi_{4}\left(x, y_{B C}\right)-\sum_{m=y}^{y_{B C}-1} \mathbf{e}_{2}^{T} \nabla \varphi_{k}(x, m)\right. \\
& \left.-\varphi_{4}\left(x_{B C}, y\right)-\sum_{m=x}^{x_{B C}-1} \mathbf{e}_{1}^{T} \nabla \varphi_{k}(m, y)\right) .(1
\end{aligned}
$$

Paths for the $\varphi_{4}(\mathbf{x})$ calculation along the first row and column of Block-1 are shown in the upper two and lower two parts of Fig. 4, respectively. With $\delta_{x}=$ $\sum_{m=y}^{x_{B C}-1} \mathbf{e}_{1}^{T} \nabla \varphi_{k}\left(m, y_{0}+1\right)-\mathbf{e}_{2}^{T} \nabla \varphi_{k}\left(x, y_{0}\right)$ and $\delta_{y}=$ $\sum_{m=y}^{y_{B C}-1} \mathbf{e}_{2}^{T} \nabla \varphi\left(x_{0}+1, m\right)-\mathbf{e}_{1}^{T} \nabla \varphi_{k}\left(x_{0}, y\right)$, we take

$$
\begin{gathered}
\varphi_{4}\left(x, y_{0}\right)=\frac{1}{2}\left(\varphi_{4}\left(x, y_{B C}\right)-\sum_{m=y_{0}}^{y_{B C}-1} \mathbf{e}_{2}^{T} \nabla \varphi_{k}(x, m)\right. \\
\left.+\varphi_{4}\left(x_{B C}, y_{0}+1\right)-\delta_{x}\right), \\
\varphi_{4}\left(x_{0}, y\right)=\frac{1}{2}\left(\varphi_{4}\left(x_{B C}, y\right)-\sum_{m=x_{0}}^{x_{B C}-1} \mathbf{e}_{1}^{T} \nabla \varphi_{k}(m, y)\right. \\
\left.+\varphi_{4}\left(x_{0}+1, y_{B C}\right)-\delta_{y}\right) .
\end{gathered}
$$

given in Fig. 5(a)-(c) and (g)-(i) for reconstruction block sizes $B C=64,32$, and 16, where significant blocking artifacts are readily apparent. Corresponding results obtained using the new algorithm given in this paper are shown in Fig. 5(d)-(f) and (j)-(1). It is clear that the new algorithm provides smooth and seamless reconstruction across block boundaries and has succeeded in eliminating virtually all of the artifacts, thereby dramatically improving both the visual quality of the reconstructions and their usefulness in assessing the accuracy of the estimated AM and FM functions.

\section{References}

[1] A. C. Bovik, M. Clark, and W. S. Geisler. Multichannel texture analysis using localized spatial filters. IEEE Trans. Pattern Anal. Machine Intell., 12(1):55-73, Jan. 1990.

[2] A. C. Bovik, N. Gopal, T. Emmoth, and A. Restrepo. Localized measurement of emergent image frequencies by Gabor wavelets. IEEE Trans. Info. Theory, 38(2):691-712, Mar. 1992.

[3] J. P. Havlicek, D. S. Harding, and A. C. Bovik. Multicomponent signal demodulation and reconstruction using AM-FM modulation models. In Proc. 1995 IEEE Workshop Nonlin. Signal and Image Proc., Neos Marmaras, Halkidiki, Greece, Jun. 20-22, 1995.

[4] J. P. Havlicek, D. S. Harding, and A. C. Bovik. The multicomponent AM-FM image representation. IEEE Trans. Image Proc., 5(6):1094-1100, Jun. 1996.

[5] J. P. Havlicek, D. S. Harding, and A. C. Bovik. Multidimensional quasi-eigenfunction approximations and multicomponent AM-FM models. IEEE Trans. Image Proc., 9(2):227242, Feb. 2000.

[6] J. P. Havlicek, P. C. Tay, and A. C. Bovik. AM-FM image models: Fundamental techniques and emerging trends. In A. Bovik, editor, Handbook of Image and Video Processing, pages 377-395. Elsevier Academic Press, Burlington, MA, 2 edition, 2005.

[7] S. Lu and P. C. Doerschuk. Nonlinear modeling and processing of speech based on sums of AM-FM formant models. IEEE Trans. Signal Proc., 44(4):773-782, Apr. 1996.

[8] P. Maragos and A. C. Bovik. Image demodulation using multidimensional energy separation. J. Opt. Soc. Amer. A, 12(9):1867-1876, Sep. 1995.

[9] R. A. Sivley and J. P. Havlicek. Multidimensional phase unwrapping for consistent APF estimation. In Proc. IEEE Int'l. Conf. Image Proc., pages 458-461, Genoa, Italy, Sep. 11-14, 2005, vol. II.

[10] R. A. Sivley and J. P. Havlicek. A spline-based framework for perfect reconstruction AM-FM models. In Proc. IEEE Southwest Symp. Image Anal., Interp., Denver, CO, Mar. 2628, 2006, to appear.

\section{Results and Discussion}

Examples of approximate AM-FM reconstructions obtained using the only previously existing algorithm [5] are 

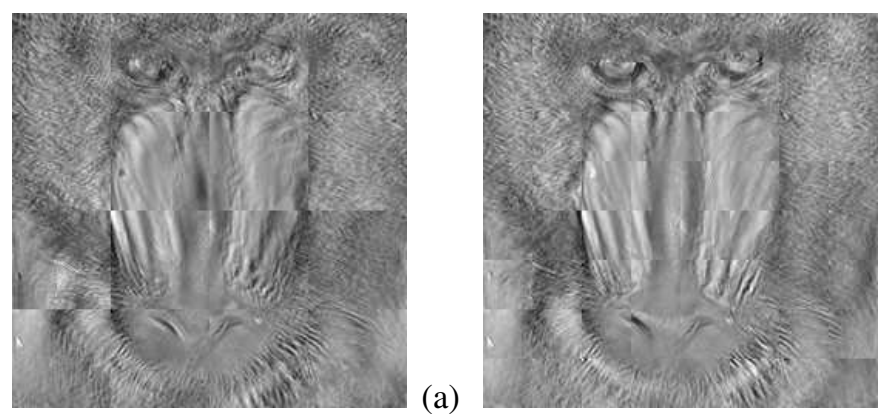

(b)
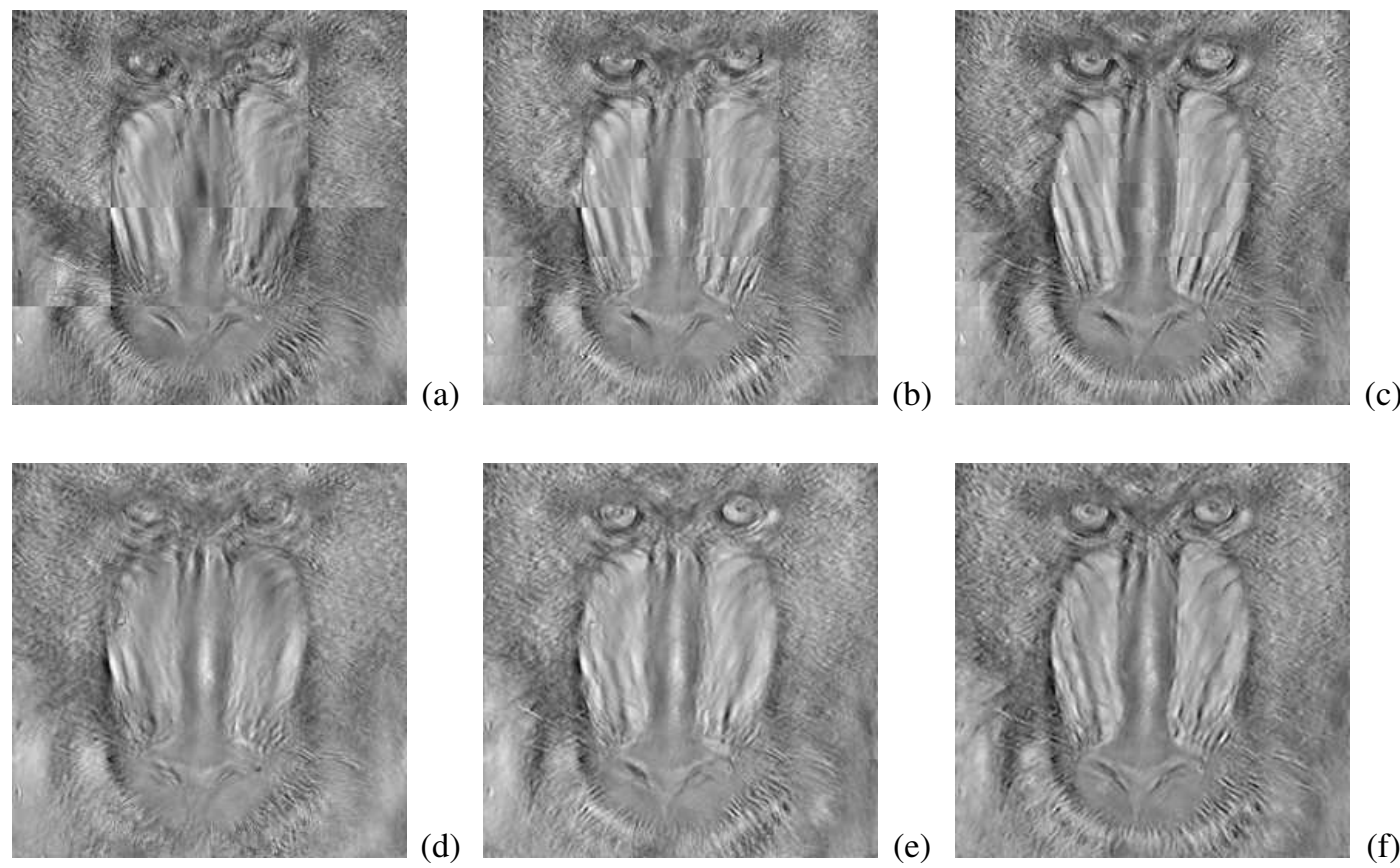

(c)
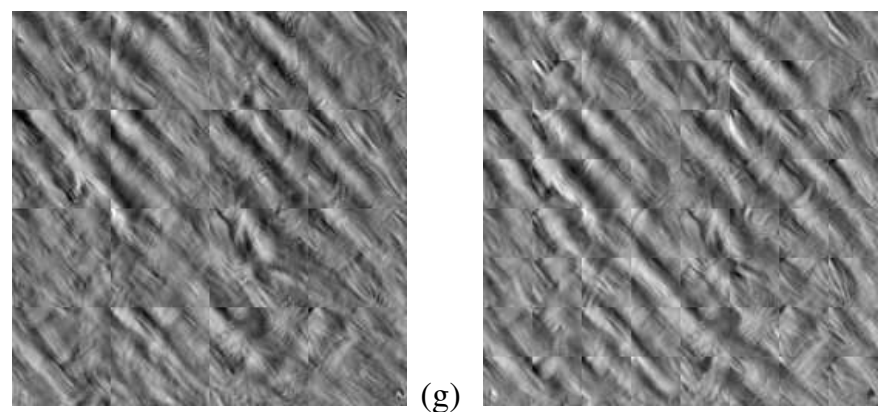

(e)
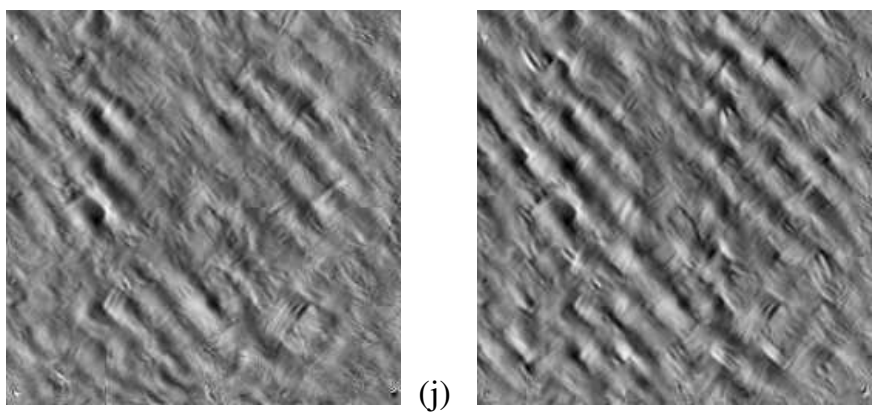

(h)

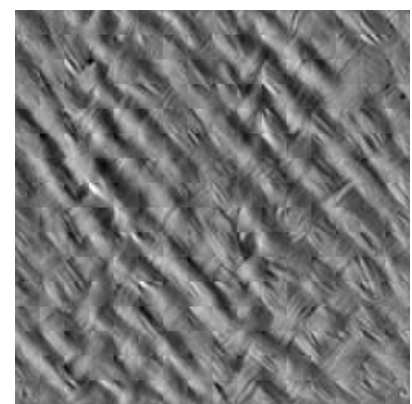

(i)

(j)

$(\mathrm{k})$

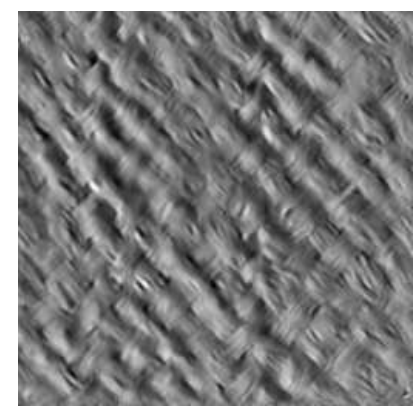

(1)

Figure 5. Approximate AM-FM image reconstructions obtained with the only previous algorithm (a)(c) and (g)-(i), which show severe blocking artifacts, and with the new proposed algorithm (d)-(f) and (j)-(I), where the blocking artifacts have been virtually eliminated. Sublattice reconstruction block sizes for these examples are $64^{2}, 32^{2}$, and $16^{2}$. 